\title{
A SEARCH FOR RADIO RECOMBINATION LINES OF POSITRONIUM NEAR THE GALACTIC CENTER
}

\author{
K. R. Anantharamaiah ${ }^{1,4}$, V. Radhakrishnan ${ }^{2}$, D. Morris ${ }^{3}$ \\ M. Vivekanand ${ }^{3,4}$, D. Downes ${ }^{3}$, C. S. Shukre ${ }^{2}$ \\ ${ }^{1}$ NRAO-VLA, P.O. Box O \\ Socorro, NM 87801, USA \\ ${ }^{2}$ Raman Research Institute \\ Bangalore 560 080, India \\ ${ }^{3}$ Institut de Radio Astronomie Millimetrique \\ Domaine Universitaire \\ 38406 St. Martin d'Heres, France
}

\begin{abstract}
Several attempts were made to detect the possible radio recombination lines of positronium near the galactic center. An absorption feature seen at $\lambda 6 \mathrm{~cm}$, in the Dconfiguration of the VLA was not confirmed by subsequent observations at $\lambda 6 \mathrm{~cm}$ and $\lambda 20 \mathrm{~cm}$ using the $\mathrm{B}$ and $\mathrm{C}$ configurations of the VLA. An observation at $\lambda 3 \mathrm{~mm}$ using the IRAM $30 \mathrm{~m}$ telescope also did not detect any line. On the basis of one recombination line photon for every positron (McClintock 1984), our non-detections imply an upper limit to the positron production rate of $<3.1 \times 10^{43} \mathrm{~s}^{-1}$, within about $2^{\prime \prime}$ of the galactic center.
\end{abstract}

\section{Introduction}

There are strong theoretical reasons for expecting positrons to be widespread: e.g. in galactic nuclei, pulsars, novae, etc. (see McClintock 1984 and references therein). An obvious signature of positrons is the $511 \mathrm{keV}$ annihilation line. This line has been observed in at least two celestial objects: solar flares (e.g. Share et al 1983) and the galactic centre (see MacCallum and Leventhal 1983). Early observations of the galactic center region suggested the presence of a time variable and therefore compact source $(<0.3 \mathrm{pc})$ (Riegler et al 1981). Because of the poor angular resolution of the gamma ray detectors the position of the point source is ill defined, but it has been postulated to be a black hole at the galactic center (See Ramaty and

4 On leave from Raman Research Institute, Bangalore, India

607

M. Morris (ed.), The Center of the Galaxy, 607-616.

(c) 1989 by the IAU. 
Lingenfelter 1987, also this volume). This line has not been detected since 1979 . Recently, Share et al (1988) have presented data from the Solar Maximum Mission (SMM) satellite, which indicate the presence of a diffuse source of galactic positrons and which can probably be accounted for by the ejecta of novae and supernovae (Signore and Vedrenne 1988).

Another signature of positrons, which should allow improved resolution, is the recombination spectrum produced when a positron and an electron combine to form Positronium (Ps). The spectrum is analogous to the hydrogen recombination spectrum and the frequencies of the different transitions can be calculated using the well known Rydberg formula. The reduced mass is now the mass of Ps $\left(\sim 2 m_{e}\right)$ and the frequencies are roughly a factor of two lower than $\mathrm{H}$ recombination lines. The optical spectrum of Ps has the analogous Lyman, Paschen and Balmer series. These have been discused by McClintock (1984).

We report here an attempt to detect the radio recombination lines of positronium, in the direction of the galactic centre, at $\lambda 6 \mathrm{~cm}$ and $\lambda 20 \mathrm{~cm}$ using the Very Large Array $^{\dagger}$ (VLA) and at $\lambda 3 \mathrm{~mm}$ using the IRAM 30-m telescope in Pico Veleta (Spain).

\section{What Can We Expect?}

Theoretical calculations and continuum measurements (Leventhal et al 1978) imply that $90 \%$ of positrons annihilate after first forming positronium. There are two competing processes which lead to the formation of Ps: (1) radiative recombination of free positrons and electrons; $e^{+}+e^{-}=P s$ and (2) charge exchange of positrons with neutral H; $e^{+}+H=p^{+}+P s$ (Bussard, Ramaty, and Drachman 1979).

If the Ps is formed in an excited state, it will radiatively decay through successive transitions to the ground state and finally annihilate via 2 photon ( $511 \mathrm{keV}$ each) emission from the singlet $S$ state or 3 photon (continuum 0 to $511 \mathrm{keV}$ ) emission from the triplet $\mathrm{S}$ state. The radiative transitions between successive excited states produces the positronium recombination lines.

The relative intensities of Ps recombination lines, to first order, can be expected to be similar to those of $\mathrm{H}$ recombination lines, given similar physical conditions. In the absence of any detailed theoretical calculations, we estimate the expected strength of the Ps recombination lines based on the simple assumption that: the fate of every positron is to form positronium in an excited state $(n>100)$ and dribble down through the different levels to the ground state and finally annihilate (McClintock 1984). As a result every positron will give rise to, for example, one photon of the Ps $87 \alpha$ line. Obviously, this is a very optimistic estimate. The luminosity in the line is therefore given by $L_{\nu}=N_{e} h \nu$ where $N_{e}+$ is the rate of production of positrons. The total flux in the line, $F_{\nu}=L_{\nu} / 4 \pi R^{2}$ and the peak

$\dagger$ VLA is part of the National Radio Astronomy Observatory, which is operated by Associated Universities Inc., under contract with the NSF 
flux in the line, $S_{\nu}=F_{\nu} / \Delta \nu$, where $\Delta \nu$ is the width of the line and $\mathrm{R}$ is the distance to the source.

Following McClintock (1984), we note that, in a photoionized region, for a wide range of temperatures $\left(<10^{6} \mathrm{~K}\right)$ and densities $\left(<10^{14} \mathrm{~cm}^{-3}\right)$, most positrons first thermalize and then form Ps by radiative recombination (Crannel et al 1976, Bussard, Ramaty, and Drachman 1979). At a given temperature Ps lines are doppler broadened 30 times more than the hydrogen lines $\left(\sqrt{m_{H} / m_{P s}}\right)$. As has been pointed out by McClintock (1984), the width is determined by the gas temperature in the annihilation region and not by the initial velocity of the positrons (which may be ultra-relativistic). At a temperature of $10000 \mathrm{~K}$ the width of the Ps $87 \alpha$ line is $\Delta \nu \sim 6.4 \mathrm{MHz}$. If the positron production rate is $N_{e^{+}}=4 \times 10^{43} s^{-1}$ (Ramaty and Lingenfelter 1987), we then expect $S_{\nu} \sim 1.7 \mathrm{mJy}$, for the peak flux of the line near $\lambda 6 \mathrm{~cm}$, with a width of $6.4 \mathrm{MHz}$. Such a line is well within detectability with the VLA. In a collisionally ionized medium, at $T<3 \times 10^{5} \mathrm{~K}$, charge exchange is the dominant formation process for Ps. At $8000 \mathrm{~K}$ the line widths are about $800 \mathrm{~km}$ $\mathrm{s}^{-1}$ (Bussard et al 1979)

Evidence for positron production near the galactic center has come from observation of the $511 \mathrm{keV}$ annihilation line prior to 1980 (see MacCallum and Leventhal 1983 for a review). An annihilation rate of $\sim 4 \times 10^{43} \mathrm{~s}^{-1}$ has been inferred from the measured flux of $\sim 2 \times 10^{-3}$ photons $\mathrm{cm}^{-2} \mathrm{~s}^{-1}$. However, the flux decreased to less than one third this value in 1981 and became undetectable after that (Leventhal et al 1986). If this variability is real, then it implies a size for the emitting region of $<10^{18} \mathrm{~cm}$ (or $\left.<7^{\prime \prime}\right)$. Recently, however, Share et al (1988) have reported data from the SMM satellite for the period 1980-1986. This has the surprising result that a nearly constant $511 \mathrm{keV}$ flux of $\sim 2 \times 10^{-3}$ photons $\mathrm{cm}^{-2} \mathrm{~s}^{-1}$ is seen every year. This has been interpreted by Share et al (1988) as evidence for a diffuse source of galactic positrons. Although the opening angle of the detectors used by the SMM and the previous balloon-borne experiments are different, the new results of SMM have weakened the evidence for a variable positron source at the galactic center.

The radio observations reported here are sensitive only to relatively compact annihilation regions.

\section{VLA Observations and Results}

We first searched for the Ps recombination lines, in June 1987, near $\lambda 6 \mathrm{~cm}$ in the D-configuration of the VLA, which has an angular resolution of about 14 arcsec. We observed in two sessions of 8 hours each at a frequency of $4915 \mathrm{MHz}$ which is near the Ps $87 \alpha$ transition $(4911 \mathrm{MHz})$. A bandwidth of $50 \mathrm{MHz}$ was used. The correlator provides 16 channels across this band. After Hanning smoothing, the resolution is $6.25 \mathrm{MHz}$ or $380 \mathrm{~km} \mathrm{~s}^{-1}$. The amplitude was calibrated by observing the source $3 \mathrm{C} 286$, which was assumed to have a flux density of $7.38 \mathrm{Jy}$. The instrumental phase was calibrated by frequent observations of the source NRAO530. 
The frequency responses of all the baselines were corrected using a single $30^{m}$ observation of the source $3 \mathrm{C} 84$, made at the end of each observing session. In order to detect any weak excess emission or absorption at the line frequency, it is necessary to subtract an average continuum from all the channels. This was done in two stages. First, a deconvolved image of the average of the central three quarters of the band was formed. The CLEAN components from the deconvolution were Fourier transformed and subtracted from the UV data of all the channels. This form of subtraction is better for large bandwidth observations, where the sidelobe structure varies from channel to channel. From the residual UV data, "dirty" channel images were constructed. Second, an average residual image formed from channels where no line was expected $(2,3,13$, and 14) was subtracted from each of the channel images. These are the final "line images". The data from both the sessions were reduced as described above and the final line images were averaged.
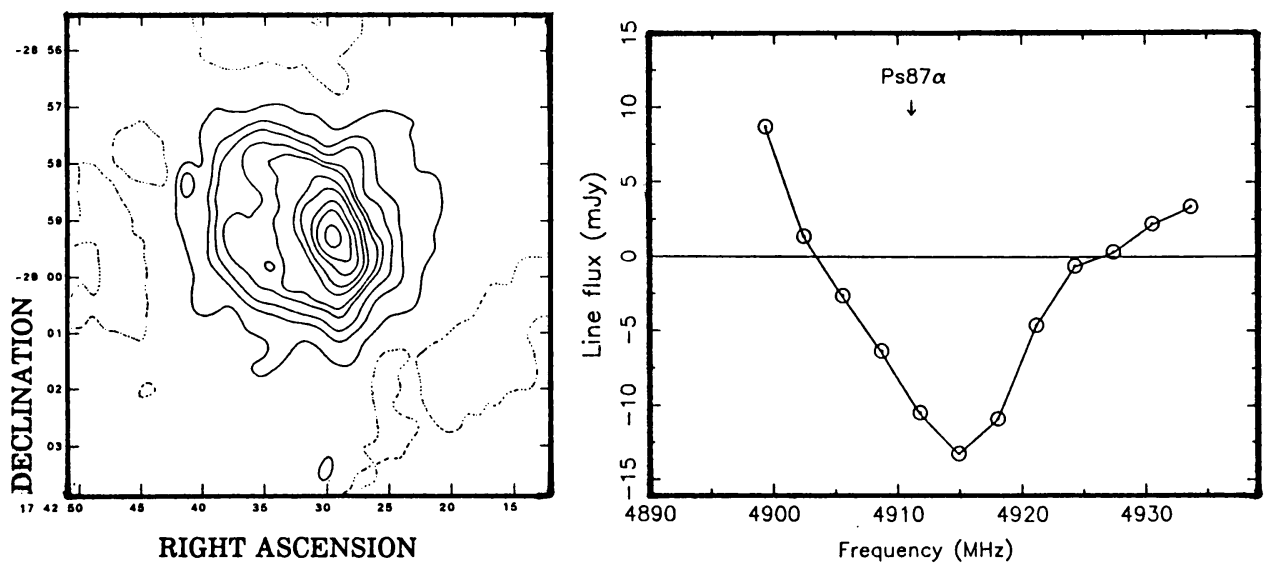

Figures 1 and 2. Continuum image of the $\mathrm{SgrA}$ region at $\lambda 6 \mathrm{~cm}$ with an angular resolution of $27^{\prime \prime} \times 15^{\prime \prime}$ and the spectrum of the Ps $87 \alpha$ line near the continuum peak. The contour levels are $-0.05, \quad 0.1,0.3,0.5,0.8,1.0,1.5,2.0,3.0,4.0$ and 6.0 $\mathrm{Jy} /$ beam.

In Fig 1, we show the continuum image of the galactic center region. A spectrum can be formed at any position on this image by taking the corresponding pixel values in the line images. One such spectrum made near the peak of the continuum is shown in Fig 2. A linear baseline fitted to the outer channels has been removed from this spectrum. Similar spectra were seen over a region of $\sim 1^{\prime}$ near the peak of the continuum.

The wide (FWHM $\sim 10.4 \mathrm{MHz}$ ) absorption feature in Fig 2 is surprising, since we expected a much weaker emission line. (Recombination lines of hydrogen are always in emission.) If this feature is real, then it implies an excitation temperature of a 
few $100 \mathrm{~K}$ for the high quantum number states of Positronium. The width of the line would represent the velocity distribution of Ps atoms and the central negative velocity, an outflow. This would be connected to the process by which Ps is formed - charge exchange or recombination. On the other hand, there is a strong possibility that the absorption feature which appears only near the peak of the continuum is an instrumental effect. The ratio of the peak of the absorption to the continuum is $1.9 \times 10^{-3}$. The signal to noise ratio of this feature is $\sim 12$. Therefore, if this feature is real, then it implies that the instrumental frequency response has been corrected to better than $0.01 \%$. Although this has been done with narrow bandwidths $(<1$ $\mathrm{MHz}$ ), it is yet to be established that it is possible with the maximum bandwith used here and in the presence of a strong complex continuum structure; the total flux in the beam is $\sim 90 \mathrm{Jy}$. Similar analysis of the data taken for the calibrator NRAO530 did not produce any feature. However, instrumental effects in this case would be much less since the source is point like and has a total flux of only $6 \mathrm{Jy}$.

In order to test the reality of the absorption feature in Fig 2, we performed several more observations described below. Since the VLA moved away from the compact D-configuration in June 1987, these follow-up observations could be done only in the larger $\mathrm{B}$ and $\mathrm{C}$ configurations.

Ps87 $\alpha(4911 \mathrm{MHz})$ and Ps89 $\alpha(4589 \mathrm{MHz})$ recombination lines were observed for 3 hours each in the B-configuration, which has an angular resolution of about 1.2 arcsec. The observing method was similar to the previous one. The instrumental frequency response was corrected using the phase calibrator NRAO530, which was observed for $\sim 8^{m}$, every $20^{m}$ or so. The continuum data was self-calibrated to remove the atmospheric phase variations and the corrections were applied to all the line channels. The line images were obtained as described before.

In Fig $3 \mathrm{~b}$, we show the continuum image of the central region of the galaxy. With this angular resolution, the emission is dominated by the compact source $\mathrm{SgrA}^{*}$. The "spiral-like" extended feature is the thermal source SgrA West located at the center of the galaxy. Spectra of the two recombination lines, made at the peak of the continuum (position of $\mathrm{SgrA}^{*}$ ) are shown in Fig 4. No obvious feature is seen in the two spectra. The $3 \sigma$ upper limit to the line strengths are $1.7 \mathrm{mJy}$ and $1.3 \mathrm{mJy}$, respectively. The latter implies a $3 \sigma$ limit to the line to continuum ratio of $1.4 \times 10^{-3}$. We should note here that, in this observation, the frequency response is corrected to much better accuracy since much of the extended continuum emission observed in the D-configuration is resolved out. Even so, the Ps $87 \alpha$ spectrum (Fig 4) shows a baseline ripple. These observations indicate that the absorption-like feature seen in the D-configuration may also be a baseline ripple or that the absorption does not occur in front of the point source $\operatorname{SgrA}^{*}$; the sensitivity to any absorption is maximum at the continuum peak. A spectrum made by averaging the line images over Sgr A West, also did not show any feature; the rms noise was $14 \mathrm{mJy}$. The RMS noise in the individual line images, made off the continuum peak, was $\sim 300 \mu \mathrm{Jy}$ and no signal was evident in any of these images. 

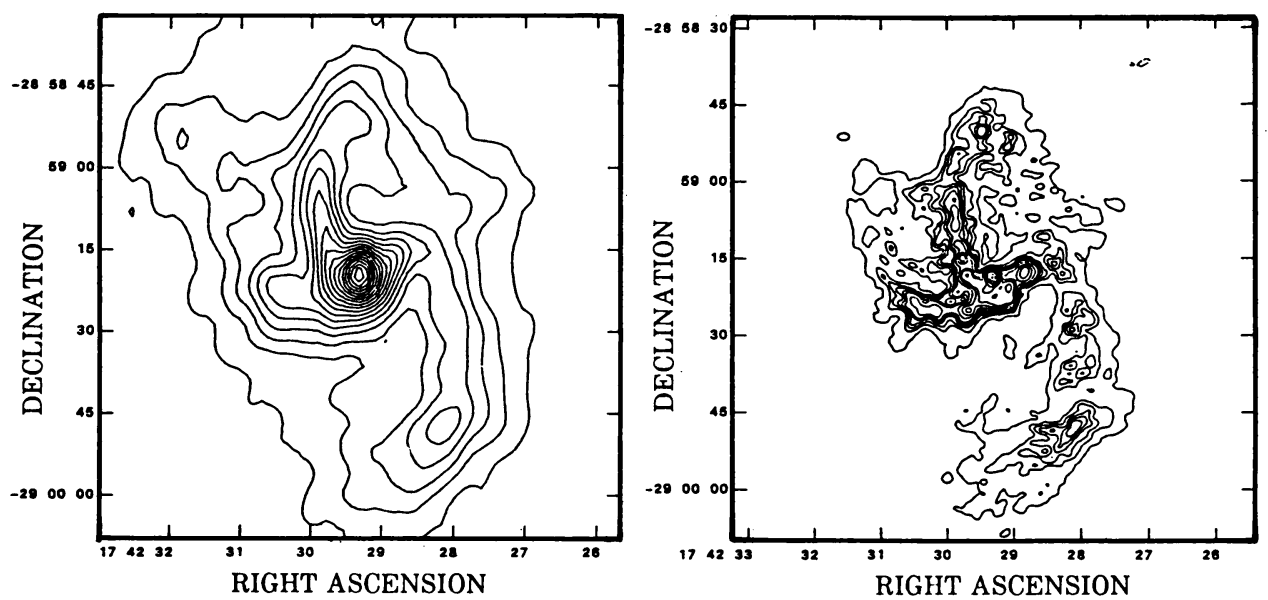

Figure 3. (a) $\lambda 6 \mathrm{~cm}$ continuum image of $\mathrm{SgrA}$ made in the $\mathrm{C}$-configuration. Resolution is $10.7^{\prime \prime} \times 7.5^{\prime \prime}$. With 1 unit $=160 \mathrm{mJy} /$ beam, the contour levels are: 1.0 to 10.0 in steps of 0.5 unit. (b) Image made in the B-configuration at $\lambda 6 \mathrm{~cm}$. Resolution $2^{\prime \prime} \times 1.6^{\prime \prime}$. With 1 unit $=4 \mathrm{mJy} /$ beam, the contour levels are: $5,8,10,12,14,16$, $20,25,30,40,50,75,100,150,200,250$ units.
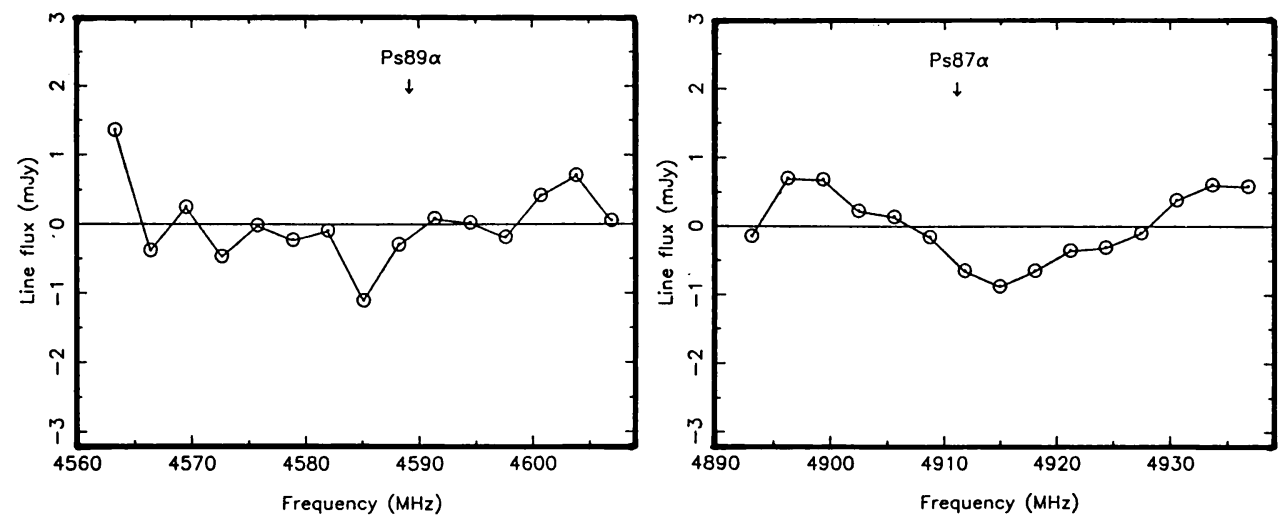

Figure 4. Spectrum of Ps89 $\alpha$ and Ps87 $\alpha$ at the peak of the continuum shown in Fig 3b. Measurements were made in the B-configuration.

Further observations were made at $\lambda 6 \mathrm{~cm}$ and $\lambda 20 \mathrm{~cm}$ in the C-configuration. At $\lambda 6 \mathrm{~cm}$ we observed the same transitions as in the B-configuration. The observing procedure and data reduction were as described before. The RMS noise in the line images was $\sim 600 \mu \mathrm{Jy}$. No signal was seen in any of the line images. The continuum emission in the central region is shown in Fig 3a. Spectra made at the peak of 
the continuum are shown in Fig 5 . These spectra are also limited by a sinusoidal baseline. The $3 \sigma$ upper limit to any line feature is $3 \mathrm{mJy}$.
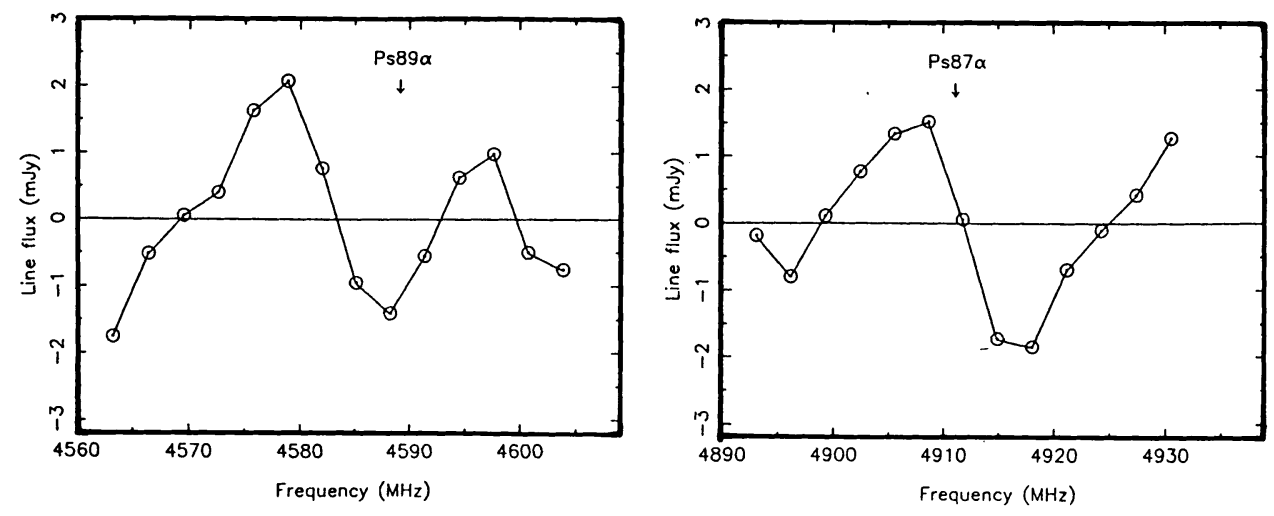

Figure 5. Spectrum of $\mathrm{Ps} 89 \alpha$ and $\mathrm{Ps} 87 \alpha$ at the peak of the continuum shown in Fig 3a. Measurements were made in the C-configuration.

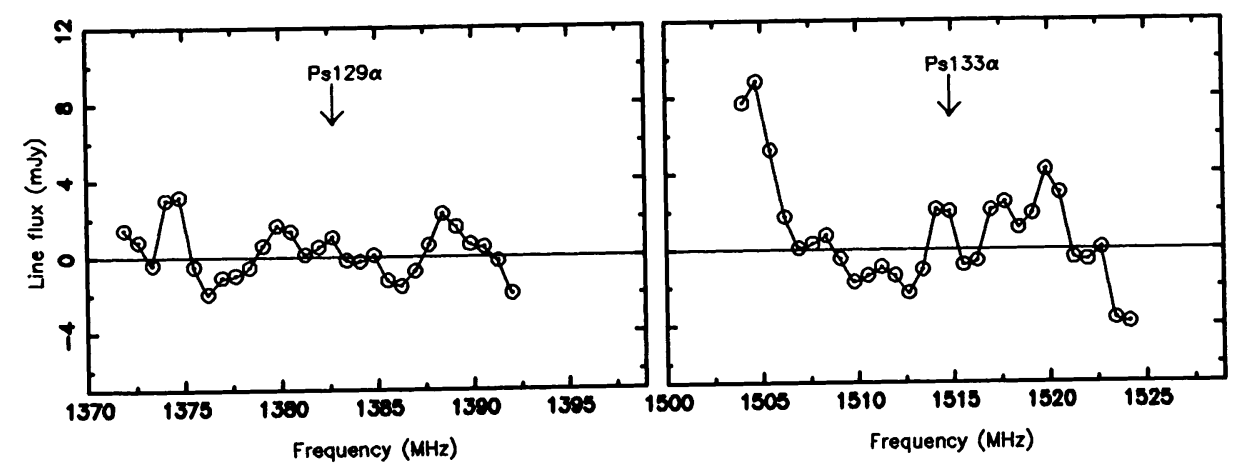

Figure 6. Spectrum of Ps133 $\alpha$ and Ps $129 \alpha$ at the peak of the continuum at $\lambda 20 \mathrm{~cm}$. Measurements were made in the C-configuration.

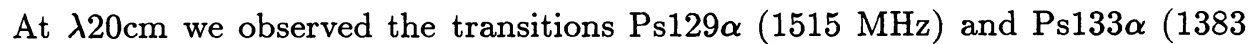
$\mathrm{MHz}$ ), using a bandwidth of $25 \mathrm{MHz}$ with 32 channels across the band. After Hanning smoothing, the velocity resolution is $\sim 310 \mathrm{~km} \mathrm{~s}^{-1}$. The sources $3 \mathrm{C} 273$, $3 \mathrm{C} 380$ and $3 \mathrm{C} 48$ were observed for $12^{\mathrm{m}}$ each and the data were used to correct the frequency response. The line images were obtained as before. The spectra at the two frequencies near the peak of the continuum are shown in Fig 6. The emission features near $1375 \mathrm{MHz}$ and $1505 \mathrm{MHz}$ in Fig 6 are due to hydrogen recombination lines. No feature due to positronium is evident with $3 \sigma$ upper limits of 3.4 and 5.1 $\mathrm{mJy}$, respectively. 
The results of all the observations using the VLA are given in Table 1. In summary, a possible Ps $87 \alpha$ absorption feature detected in the D-configuration was not confirmed by subsequent observations of Ps87 $\alpha$ and Ps89 $\alpha$ lines in the B and $\mathrm{C}$ configurations and Ps $129 \alpha$ and Ps133 $\alpha$ in the C-configuration. All the spectra obtained here are limited by baseline uncertainities. It is perhaps desirable to repeat the $\mathrm{D}$-configuration observations near another transition, particularly when the baseline ripple problem is better understood.

Table 1: Summary of VLA observations

\begin{tabular}{|c|c|c|c|c|c|c|}
\hline Date & Line & $\begin{array}{l}\text { Configuration } \\
\text { and } \\
\text { Wavelength } \\
\end{array}$ & $\begin{array}{l}\text { beam } \\
\text { "x" }\end{array}$ & $\begin{array}{c}\text { Peak } \\
\text { continuum } \\
\mathrm{Jy}\end{array}$ & $\begin{array}{l}\text { Peak } \\
\text { Line } \\
\text { mJy }\end{array}$ & $\begin{array}{c}\text { Line to } \\
\text { continuum } \\
\text { ratio }\left(\times 10^{-3}\right)\end{array}$ \\
\hline June 87 & $\operatorname{Ps} 87 \alpha$ & D $6 \mathrm{~cm}$ & $27 \times 15$ & 7.10 & $\leq-13.3 \pm 3$ & $\leq-1.9 \pm 0.4$ \\
\hline Nov 87 & $\begin{array}{l}\text { Ps } 87 \alpha \\
P s 89 \alpha\end{array}$ & B $6 \mathrm{~cm}$ & $\begin{array}{l}2.0 \times 1.6 \\
3.5 \times 1.6\end{array}$ & $\begin{array}{l}0.85 \\
0.91\end{array}$ & $\begin{array}{l}<1.7 \\
<1.3\end{array}$ & $\begin{array}{l}<2.0 \\
<1.4\end{array}$ \\
\hline Jan 88 & $\begin{array}{l}\text { Ps } 129 \alpha \\
\text { Ps } 133 \alpha\end{array}$ & C $20 \mathrm{~cm}$ & $\begin{array}{l}10.7 \times 7.5 \\
12.2 \times 6.1\end{array}$ & $\begin{array}{l}3.04 \\
2.95\end{array}$ & $\begin{array}{l}<5.1 \\
<3.4\end{array}$ & $\begin{array}{l}<1.7 \\
<1.2\end{array}$ \\
\hline Mar 88 & $\begin{array}{l}\text { Ps } 87 \alpha \\
\text { Ps } 89 \alpha\end{array}$ & C $6 \mathrm{~cm}$ & $\begin{array}{l}8.6 \times 4.6 \\
9.1 \times 5.0\end{array}$ & $\begin{array}{l}2.40 \\
2.70\end{array}$ & $\begin{array}{l}<3.2 \\
<3.4\end{array}$ & $\begin{array}{l}<1.3 \\
<1.3\end{array}$ \\
\hline
\end{tabular}

Upper limits are $3 \sigma$.

\section{Pico Veleta Observations and Results}

Theoretical considerations following the possible detection of the line in absorption indicated that the absorption might become stronger at higher frequencies (lower quantum levels). This can happen if positronium is formed mainly by charge exchange with neutral hydrogen atoms in a collisionally ionized medium (Bussard et al 1979). To test this, we searched for the Ps $32 \alpha$ line $(95.828 \mathrm{GHz})$ within $1^{\prime}$ of the galactic center using the IRAM 30-m telescope in Pico Veleta (Spain). This particular transition was chosen from amongst the many falling in the $3 \mathrm{~mm}$ waveband because this area of the spectrum contains few confusing molecular lines.

The telescope beam $\left(26^{\prime \prime}\right)$ was centered on the continuum source and spectra were obtained using reference positions offset by 1 arcmin. Two observing methods were used. In May 1988, position switching of the telescope was used with the reference offset to positive declination. Higher quality spectra were obtained in July 1988 by "wobbling" the secondary mirror at $1 \mathrm{~Hz}$. In this case, two reference positions 1 arcmin in azimuth - on either side of the galactic center source were used. Observations were restricted to within 2 hours of meridian transit so that the reference positions rotated little in the equatorial coordinate system. The instrumental baseline, amounting to about $1 \%$ of the continuum, was measured on Saturn and subtracted. The $512 \times 1 \mathrm{MHz}$ filter bank spectrometer was used. It covers a velocity range of abouut $1600 \mathrm{~km} \mathrm{~s}^{-1}$ at $95 \mathrm{GHz}$. 
The July 1988 data is shown in Fig 7 after smoothing to a resolution of $10 \mathrm{MHz}$ $\left(30 \mathrm{~km}^{-1}\right)$. The baseline quality in this data was better than the May 1988 data. An absorption feature can be seen at $-210 \mathrm{~km} \mathrm{~s}^{-1}$. This is probably due to emission by acetaldehyde in one of the reference regions. (Much larger features $(0.5 \mathrm{~K})$ are observed with offsets of $+2^{\prime}$ in right ascension or $-2^{\prime}$ in declination.)

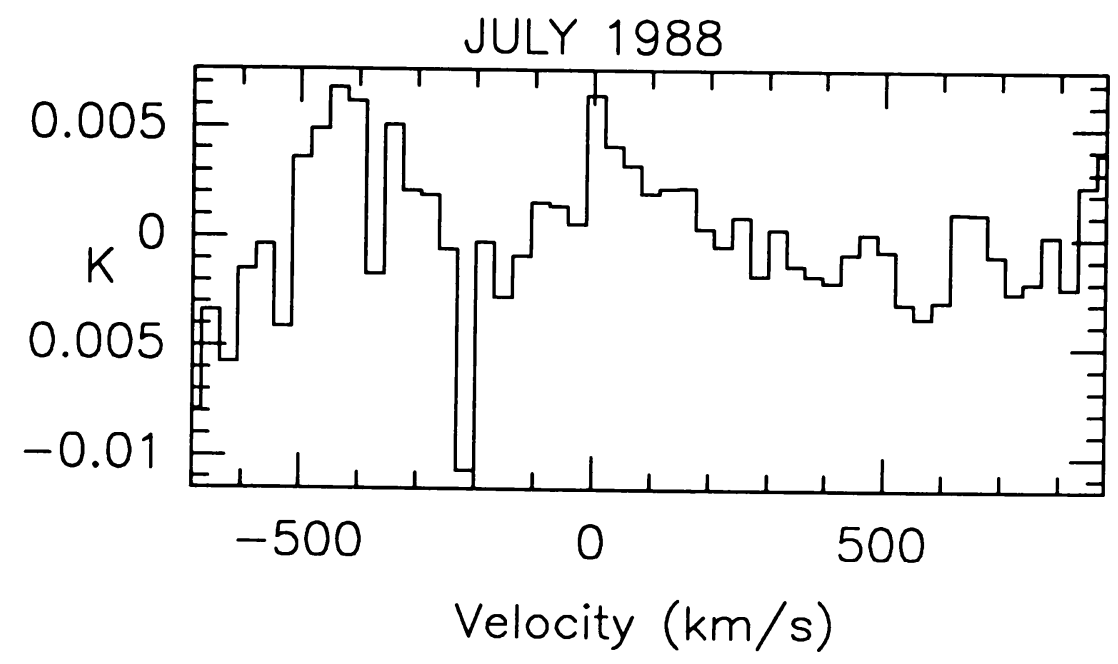

Figure 7. Spectrum made near the frequency of Ps $32 \alpha$ line using the IRAM $30 \mathrm{~m}$ telescope. A linear baseline has been removed. The integration time was 6.5 hours and the RMS is $3.3 \mathrm{mK}$.

Formal fits of Gaussian line profiles, centered at $50 \mathrm{~km} \mathrm{~s}^{-1}$, yield the intensities and RMS uncertainties listed in table 2 - for two postulated half power widths. The continuum temperature was $1.2 \mathrm{~K}$. Note that the fits all give emission features. No positive detection was made and the values in Table 2 can only be used as a guide to establish upper limits to emission lines of various widths.

Table 2: Upper limits to Ps32 $\alpha$ line

\begin{tabular}{ccc}
\hline & July data & May data \\
Width $\left(\mathrm{km} \mathrm{s}^{-1}\right)$ & Temperature $(\mathrm{mK})$ & Temperature $(\mathrm{mK})$ \\
\hline 400 (recombination) & $1.6 \pm 0.7$ & $2.3 \pm 0.9$ \\
800 (charge exchange) & $0.7 \pm 0.4$ & $1.3 \pm 0.6$ \\
\hline
\end{tabular}




\section{Conclusions}

A search for recombination lines of positronium near the galactic center at 4911 $\mathrm{MHz}$ (Ps87 $\alpha$ ) using the D-configuration of the VLA yielded a possible detection of the line, in absorption, with an optical depth of $1.9 \times 10^{-3}$ and a line width of $\sim 10.4 \mathrm{MHz}$. However, subsequent observations, at $\lambda 6 \mathrm{~cm}$ and $\lambda 20 \mathrm{~cm}$, in the $\mathrm{B}$ and C configurations of the VLA did not confirm this line - the $3 \sigma$ upper limits to the optical depths are in the range $1.3-2.0 \times 10^{-3}$. A search for the Ps32 $\alpha$ line $(95.828$ $\mathrm{GHz}$ ) using the IRAM 30-m telescope also did not yield any detection. If we assume that every positron that is produced gives rise to one photon of the recombination line (McClintock 1984), then our best $3 \sigma$ upper limit to the line strength $(1.3 \mathrm{mJy}$ at $\lambda 6 \mathrm{~cm}$, see Table 1) implies a limit for the positron production rate near the galactic center $N_{\mathrm{e}^{+}}<3.1 \times 10^{43} \mathrm{~s}^{-1}$. This refers to a region of diameter $\sim 2^{\prime \prime}$ or 0.1 pc. The limits for larger areas (20" diameter) are an order of magnitude larger.

Thus, although our observations do not provide any evidence for a point source of positrons at the galactic center, they do not conflict with the evidence from gamma ray measurements. A recent analysis suggested a maximum annihilation rate of $1.3 \times 10^{43} \mathrm{~s}^{-1}$ (Ramaty and Lingenfelter 1987). Our present limits are set by instrumental effects and it may be possible to improve them when the baseline ripples are better understood.

Acknowledgements. We thank R.D. Ekers and B.G. Clark for their encouragement and keen interest in these observations.

\section{REFERENCES}

Bussard, R.W., Ramaty, R., Drachman, R.J., 1979, Ap. J, 228, 928

Crannell, C.J., Joyce, G., Ramaty, R., Werntz, C., 1976, Ap. J, 210, 582

Leventhal, M., MacCallum, C.J., Stang, P.D., 1978, Ap. J. Lett, 225, L11

Leventhal, M., MacCallum, C.J., Huters, A.F., Stang, P.D., 1986, Ap. J, 302, 459

MacCallum, C.J., Leventhal, M., 1983, in Positron-electron pairs in

Astrophysics, ed. M. Burns, A. Harding, R. Ramaty (New York: AIP) p211

McClintock, J.E., 1984, Ap. J, 282, 291

Ramaty, R., Lingenfelter, R.E., 1987, in The Galactic Center, ed. D.C. Backer

(New York: AIP) p51

Riegler, G.R., Ling, J.C., Mahony, W.A., Wheaton, W.A., Willet, J.B., Jacobson, A.S., 1981, Ap. J, 248, L13

Share, G.H., Chupp, E.L., Forrest, D.J., Rieger, E., 1983, in Positron-electron pairs in Astrophysics, ed. M. Burns, A. Harding, R. Ramaty (New York: AIP) p15

Share, G.H., Kinzer, R.L., Kurfess, J.D., Messina, D.C., Purcell, W.R.,

Chupp, E.L., Forrest, J., Reppin, C., 1988, Ap. J, 326, 717

Signore, M., Vedrenne, G., 1988, Astron. Astrophys, 201, 379 